Article

\title{
miR-3687 Overexpression Promotes Bladder Cancer Cell Growth Through Inhibiting the Negative Effect of FOXP1 on Cyclin E2 Transcription
}

\author{
Qipeng Xie ${ }^{1,2, \#, ~ C a i y i ~ C h e n ~}{ }^{1, \#, ~ H a i y i n g ~ L i ~}{ }^{1, \#, ~ H u i y i n g ~ Y a n}{ }^{1}$, Jiheng $\mathrm{Xu}^{1}$, Dapang Rao ${ }^{2}$, Huxiang \\ Zhang ${ }^{3}$, Honglei Jin ${ }^{1}$, Haishan Huang ${ }^{1, *}$ and Chuanshu Huang ${ }^{4, *}$
}

1 Zhejiang Provincial Key Laboratory for Technology and Application of Model Organisms, Key Laboratory of Laboratory Medicine, Ministry of Education, School of Laboratory Medicine and Life Sciences, Wenzhou Medical University, Wenzhou, Zhejiang 325035, China; pandon2002@163.com (Q.X.); cychenwmu@163.com (C.C.); lihaiying0610@163.com (H.L.); m15957790178@163.com (H.Y.); 969864545@qq.com (J.X.); jhlbmd@163.com (H.J.); haishan_333@163.com (H.H.)

2 The Second Affiliated Hospital \& Yuying Children's Hospital of Wenzhou Medical University, Wenzhou, Zhejiang 325035, China; surgeon987@126.com (D.R.)

3 Biobank of Central Laboratory, the First Affiliated Hospital of Wenzhou Medical University, Wenzhou, Zhejiang 325035,China.; 383951930@qq.com (H.Z.)

4 Nelson Institute of Environmental Medicine, New York University School of Medicine, Tuxedo, NY 10987, USA; Chuanshu.huang@nyulangone.org (C.H.)

\# These authors contributed equally to this work.

* Correspondence: haishan_333@163.com; Chuanshu.huang@nyulangone.org Tel.: +01-646-754-9457

\begin{abstract}
Cyclin E2, a member of the cyclin family, is a key cell cycle-related protein. This protein plays essential roles in cancer progression, and as such, an inhibitor of cyclin E2 has been approved to treat several types of cancers. Even so, mechanisms underlying how to regulate cyclin E2 expression in cancer remain largely unknown. The current study found that miR-3687 is upregulated in clinical bladder cancer (BC) tumor tissues, TCGA database and human BC cell lines. Inhibition of miR-3687 expression significantly reduces human BC cell proliferation in vitro and tumor growth in vivo, which are concurrently with the induction of G0/G1 cell cycle arrest and downregulation of cyclin E2 protein expression. Interestingly, overexpression of cyclin E2 reversed the inhibition of BC proliferation induced by miR-3687. Mechanistic studies suggest that miR-3687 could bind to the 3'-UTR of foxp1 mRNA, downregulates FOXP1 protein expression, and in turn promotes the transcription of cyclin E2, thereby promoting the growth of $\mathrm{BC}$ cells. Collectively, the current study not only establishes a novel regulatory axis of miR-3687/FOXP1 in regard to regulation of cyclin E2 expression in BC cells, but also provides strong suggestive evidence that miR-3687 and FOXP1 may be potentially promising targets in therapeutic strategies of human BC.
\end{abstract}

Keywords: miR-3687, FOXP1, cyclin E2, Bladder Cancer, Tumor formation

\section{Introduction}

Cyclin E2 is a protein encoded by the human CCNE2 gene at $8 \mathrm{q} 22.1$ and is mostly regarded as being functionally redundant with cyclin E1 [1,2]. Both cyclin E1 and cyclin E2 display high sequence similarity (69.3\% in Homo sapiens) and important functional motifs are conserved. The latter include domains for Cdk (cyclin-dependent kinase) and Cdk inhibitor interaction, a nuclear localization sequence, and a centrosome localization sequence [3]. The principal function of cyclin E2 is to help cells switch from G0/G1 to $S$ phase by binding of CDK2 and phosphorylation of Rb protein $[4,5]$. Overexpression of cyclin E2 has been reported in many types of human cancers, including leukemia [6], and breast cancer [7,8], lung cancer [9], ovarian cancer [10], nasopharyngeal carcinoma[11], and 
bladder cancer [3,12]. In many types of cancer, overexpression of cyclin E2 correlates with tumor progression and mortality $[3,13]$. Additionally, dysregulation of cyclin E2 leads to chromosomal instability, suggesting that aberrant cyclin E2 expression may contribute to tumorigenesis oncogenic transformation, and cancer cell proliferation [14]. The cell cycle-dependent transcription of cyclin E2 is mediated by E2F transcription factors [15]. In the studies reported here, we found that the expression of cyclin $\mathrm{E} 2$ is regulated by $\mathrm{miR}-3687$ in an indirect manner.

MicroRNA (miRNA) is a class of highly conserved, non-coding small molecular containing 1925 nucleotides [16]. miRNAs are involved in the regulation of genes associated with cancer development and progression, and therefore can act as oncogenes, contributing to tumor formation $[17,18]$ or as tumor suppressors by inhibiting the expression of oncogenes in different types of cancer $[19,20]$. Many studies have shown that dysregulation of miRNAs plays a vital role in multiple biological processes associated with bladder cancer (BC), including differentiation, proliferation, cell cycle arrest, apoptosis, and metastasis [21-23]. For example, our group has identified dysregulated miRNAs (e.g., miR-411) that are involved in BC cell function. Based on this information [24]. Thus, the identification of an unknown dysregulated miRNA in human BC may provide valuable information for defining new biomarkers for BC prognosis and/or new targets for therapeutic paradigms. The current studies found that miR-3687 expression is altered in human BC tissues and cell lines. We further explore the potential biological role and mechanisms underlying the miR-3687 regulation of human bladder tumorigenicity.

\section{Results}

\section{1. miRNA-3687 was upregulated in human BC, and inhibition of miR-3687 expression suppressed cell} growth with induction of G0/G1 arrest in human $B C$ cells.

The analyses of RNA-Seq data of 19 BC available in the TCGA database showed upregulation of miR-3687 in human BC tissues in comparison to their paired normal bladder tissues ( $n=19$, Figure 1A, $\mathrm{p}$ <0.05). miR-3687 expression levels in $19 \mathrm{BC}$ tissues were compared with those in paired normal tissues obtained from the TCGA BC database and results expressed as $\log 2$ fold-change (Figure S1). Consistently, miR-3687 levels human invasive BC cell lines (e.g., UMUC3, T24, T24T, J82, TCCSUP) were higher than that in normal urothelial cell lines (UROtsa) (Figure 1B).

To evaluate the effects of miR-3687 on the regulation of human BC, the miR-3687 sponge was stably transfected into UMUC3 and T24 cells. Stable transfectants UMUC3(miR-3687i) and T24(miR3687i) and corresponding control vector transfectants were established (puromycin selection and real-time qPCR; Figures 1C and 1D). Soft-agar assay data indicated that inhibition of miR-3687 expression led to a dramatic decrease in $\mathrm{BC}$ cell anchorage-independent growth (Figures 1E and 1F; $\mathrm{p}<0.05$ ). Moreover, cell proliferation (using ATP assay) showed that monolayer BC cell growth ability was also lower in the UMUC3(miR-3687i) and T24(miR-3687i) transfectants than in their corresponding control vector transfectants, especially at a later time point day 5 (Figures $1 \mathrm{G}$ and $1 \mathrm{H}$; $\mathrm{p}<0.05)$.

To explore the underlying mechanisms of any pro-cancerous activity of miR-3687, any effect on cell cycle progression regulation was determined by flow cytometry in the UMUC3 and T24 cells. As shown in Figures 1I and 1J, inhibition of miR-3687 expression induced significant G0/G1 arrest, suggesting G0/G1 phase reduction might be associated with any promoting effect of miR-3687 in human BC cells. Together, these results suggested that miR-3687 could promote BC cell growth in vitro. 

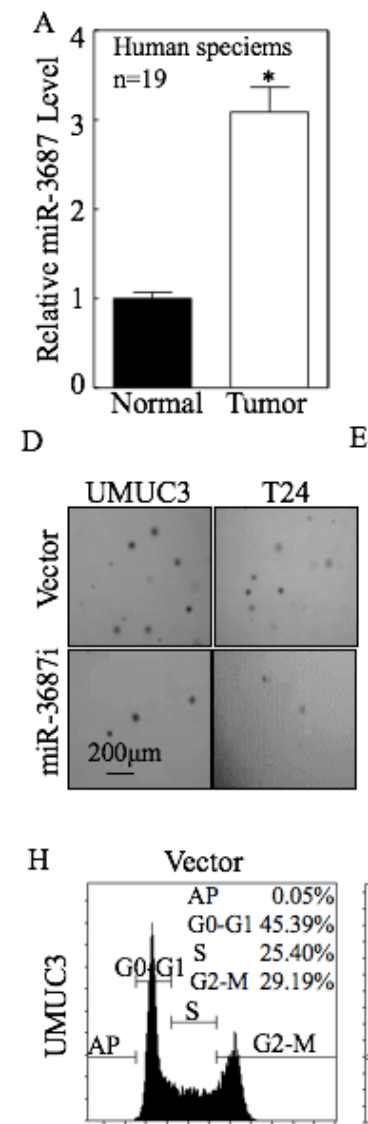
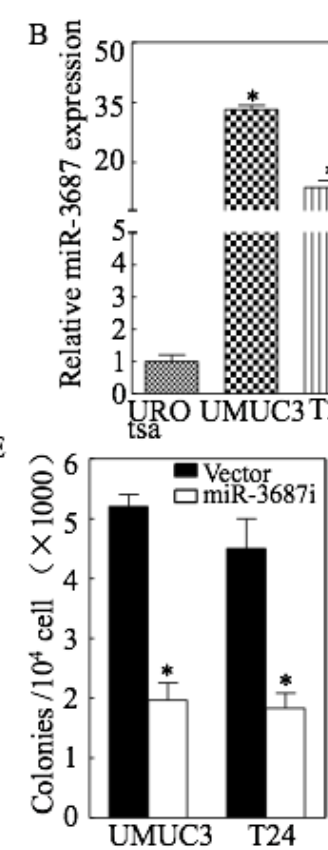

miR-3687i

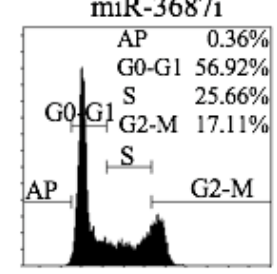

$\mathrm{C}$
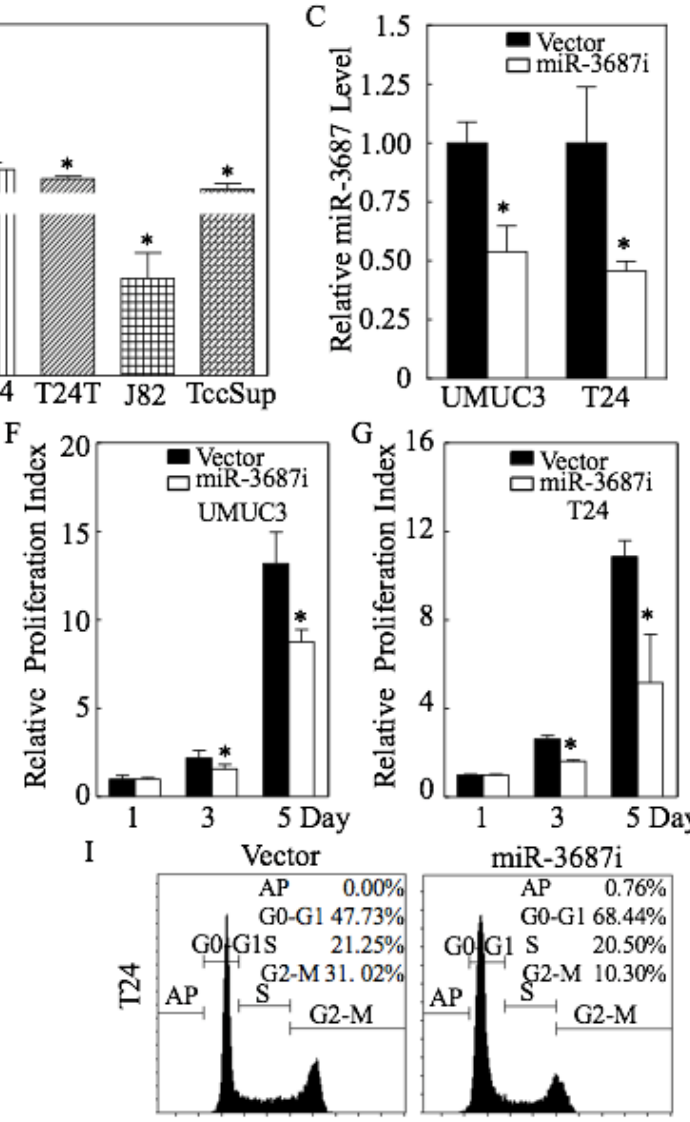

Figure 1. miR-3687 overexpression in human BC samples and BC cell lines and its role in BC cell growth and cell cycle progression. (A) miR-3687 expression levels in fresh BC tissues were compared with paired normal tissues. (B) miR-3687 levels in human BC cell lines (UMUC3, T24, T24T, J82, TCCSUP) were determined and compared with normal urothelial cell lines (UROtsa) by real-time PCR. miR-3687 expression was normalized to U6 expression. Bars represent means \pm SD. * Significant change relative to control group $(\mathrm{p}<0.05)$. ( $\mathrm{C}$ and $\mathrm{D})$ shRNA targeted miR-3687 and control vector plasmids were stably transfected into UMUC3 (C) and T24 (D) cells; stable transfectants were identified by real-time PCR. Bars represent the mean \pm SD. *Significant decrease relative to control vector cells $(p<0.05)$. ( $E$ and $F)$ Soft agar assay was used to determine the effect of miR-3687 downexpression on UMUC3 and T24 anchorage-independent growth. Representative images of colonies in indicated cells were captured by microscopy after $3 \mathrm{wk}$ of incubation. ( $\mathrm{G}$ and $\mathrm{H}$ ) Effect of miR$3687 \mathrm{i}$ on monolayer proliferative rates was evaluated by ATP assays. Results presented as means \pm $\mathrm{SD}$; * significant increase relative to vector control cells $(\mathrm{p}<0.05)$. (I and J) Indicated cells were seeded into 6-well plates and cultured to $70-80 \%$ confluence; after synchronization, cells were cultured in complete medium for another $24 \mathrm{~h}$ and then subjected to cell cycle analysis by flow cytometry.

\subsection{Inhibiting of miR-3687 expression repressed tumor growth in vivo in xenograft nude mouse}

To extend the in vitro findings that miR-3687 might promote BC cell growth to an in vivo scenario, a xenograft tumorigenic nude mouse model was employed to examine the effect of miR-3687 on the tumorigenicity of UMUC3 and T24 cells. The results showed that reduced miR-3687 expression significantly attenuated tumor growth as compared with scrambled vector control cells $(p<0.05$; Figures 2A-2F). 

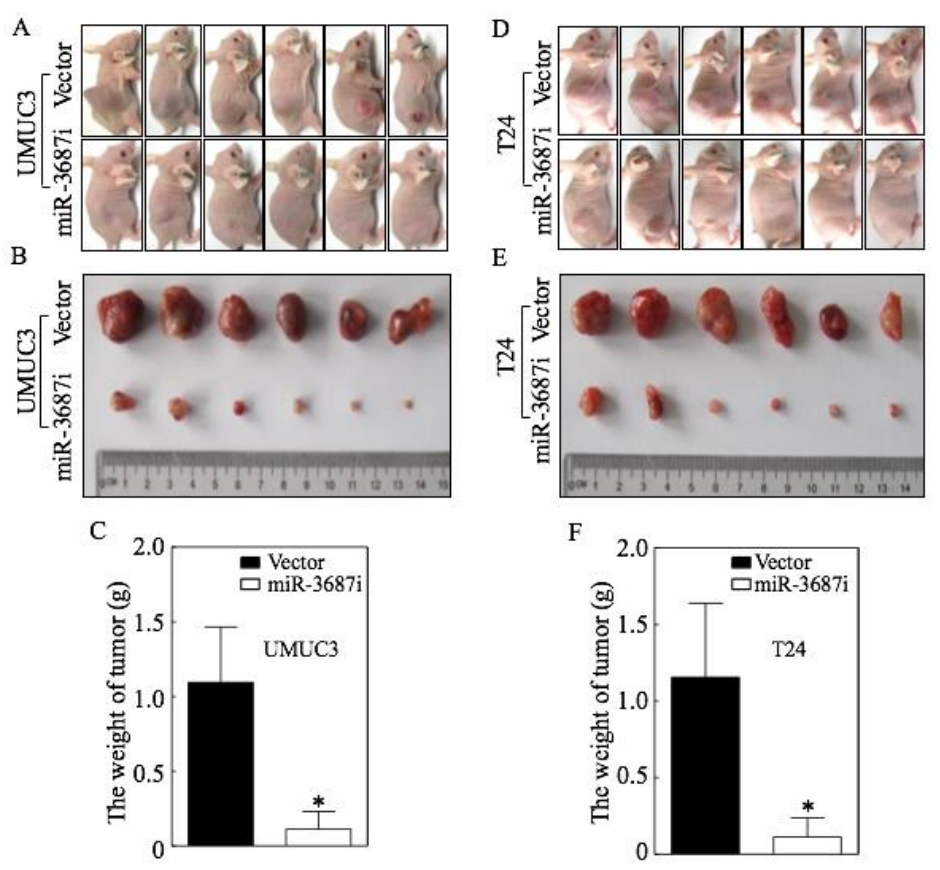

Figure 2. Effects of miR-3687 expression on tumor growth in xenograft nude mouse. (A-F) Female BALB/c athymic nude mice were subcutaneously injected with UMUC3(miR-3687i) or T24(miR3687i) cells or their vector control transfectants. The mice were euthanized after 4-5 wk, and any tumor present removed, imaged, and weighed. Results presented as means $\pm \mathrm{SD} ;{ }^{*}$ significant decrease relative to vector control cells $(p<0.05)$.

\section{3. miR-3687 promoted BC cell growth by up-regulating cyclin E2}

To further elucidate the mechanisms leading to G0/G1 arrest induced by inhibition of miR-3687 in BC cells, we examined modulators that have a significant effect on the G0 / G1 phase-related cell cycle. The results showed that inhibition of miR-3687 expression did not affect expression of cell cycle regulators CDK2, CDK4, CDK6, cyclin D1, or p21, but led to a remarkable decrease in cyclin E2 expression in the UMUC3(miR-3687i) and T24(miR-3687i) transfectants in comparison to their corresponding scramble vector transfectants (Figure 3A). When cyclin E2 was overexpressed in UMUC3(miR-3687i) cells (Figures 3B), reversed the inhibition of miR-3687 on the monolayer growth and anchorage-independent growth (Figures 3C, 3D, 3F). Flow cytometry also indicated UMUC3(miR-3687i/cyclin E2) exhibited a reverse effect of miR-3687i on inducing G0/G1 cell cycle arrest (Figure 3E). The results suggested that inhibition of cyclin E2 contributes to miR-3687imediated G0/G1 cell cycle arrest and reduction of anchorage-independent growth in BC cells. 

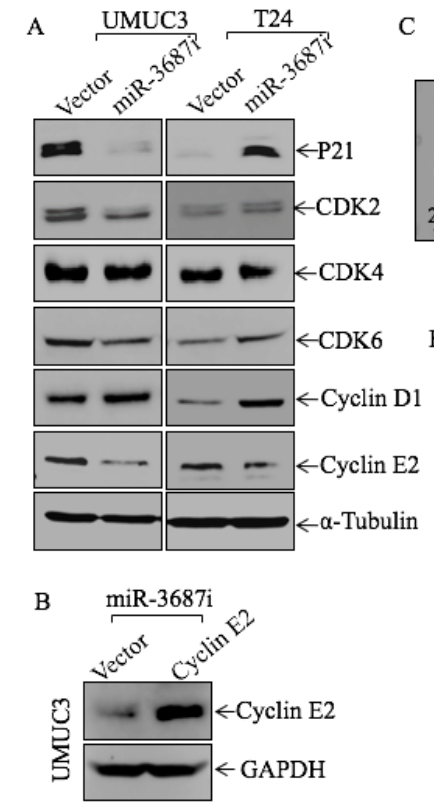
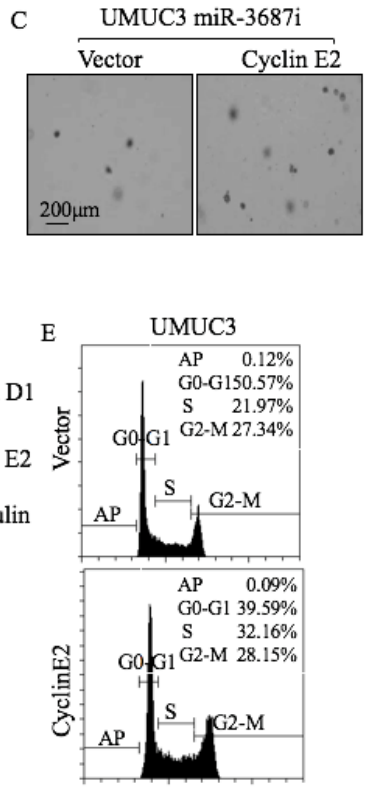
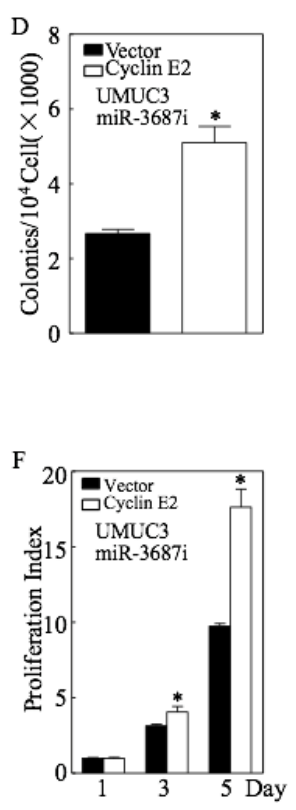

Figure 3. miR-3687 promoted BC cell growth by up-regulating cyclin E2. (A) Cell lysates from indicated cells were evaluated for p21, CDK2, CDK4, CDK6, cyclin D1, and cyclin E2 protein expression via Western blots. ๑-Tubulin served as a loading control. (B) Cyclin E2 and vector control plasmids were transfected into UMUC3(miR-3687i) cells and cyclin E2 overexpression was identified by Western blot. (C and D) Soft agar assay was used to determine the effect of cyclin E2 overexpression on anchorage-independent growth of UMUC3(miR-3687i). (E) Indicated cells were seeded in 6-well plates and cultured to $70-80 \%$ confluence; after synchronization, cells were cultured in complete medium another $24 \mathrm{~h}$ and then subjected to cell cycle analysis. (F) Effect of cyclin E2 on monolayer proliferative rates of UMUC3(miR-3687i) cells was evaluated by ATP assay. Results presented as means \pm SD. *Significant change relative to vector control cells $(\mathrm{p}<0.05)$.

\subsection{FOXP1 was responsible for miR-3687 up-regulating of cyclin E2 at the transcriptional level}

To ascertain how miR-3687 up-regulated cyclin E2, cyclin E2 mRNA levels were assessed. It was seen that cyclin E2 mRNA expression was profoundly down-regulated in UMUC3(miR-3687i) and T24(miR-3687i) transfectants in comparison to corresponding scramble vector transfectants (Figure $4 \mathrm{~A})$. To test whether miR-3687 regulates cyclin E2 mRNA transcription, the cyclin E2 promoterdriven luciferase reporter was used to compare promoter transcription activity in both cells. As seen in Figure 4B, promoter-driven luciferase reporter transcription activity in UMUC3(miR-3687i) and T24(miR-3687i) transfectants was significantly lower than scramble vector transfectants. This reflects the possibility that miR-3687 positively regulates cyclin E2 mRNA transcription. To test this, TFANSFAC ${ }^{\circledR}$ Transcription Factor Binding Sites Software (Biological Database, Wolfenbüttel, Germany) was used for bioinformatic analysis of the cyclin E2 promoter. The results indicated the cyclin E2 promoter region contains a putative DNA-binding site of OCT2, CDX2, and FOXP1 (Figure 4C). Therefore, this study next determined the effect of miR-3687i on the expression of transcription factors in human BC cells.

As shown in Figure 4D, increasing FOXP1 protein expression was seen in UMUC3(miR-3687i) and T24(miR-3687i) transfectants compared to the scramble vectors. There was no marked change in OCT2 and CDX2 expression. FOXP1 is a member of the FOX family of transcription factors and specifically acts as a transcriptional repressor for its downstream-regulated gene [25]. FOXP1 regulates a variety of important developmental aspects, including tissue development in the lung, brain, thymus, and heart [26]. Thus, we anticipate that FOXP1 might play a role in miR-3687i inhibiting cyclin E2 transcription in human BC cells. 
A

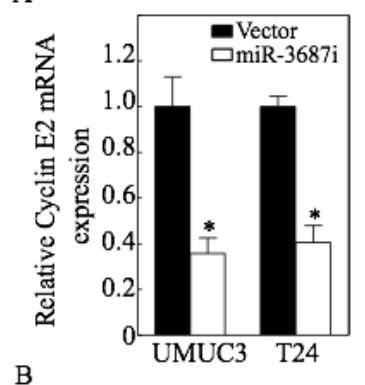

$\mathrm{B}$

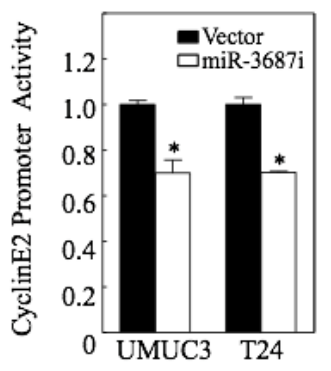

$\mathrm{C}$

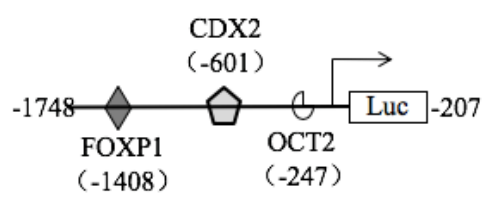

D

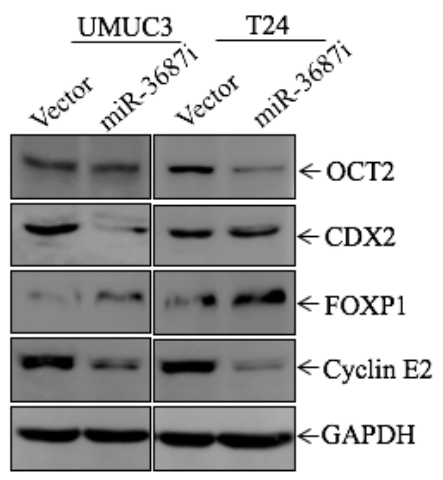

Figure 4. Inhibition of miR-3687 attenuated cyclin E2 transcription with upregulating FOXP1 protein. (A) Relative Cyclin E2 mRNA expression detected in UMUC3(miR-3687i) and T24(miR-3687i) was compared to their vector control cells. (B) UMUC3(Vector) vs. UMUC3(miR-3687i) cells and T24(Vector) vs. T24(miR-3687i) cells were transiently transfected with a cyclin E2 promoter-driven luciferase reporter together with pRL-TK. Transfectants were seeded into 96-well plates to determine cyclin E2 promoter transcriptional activity. pRL-TK used as internal control to normalize transfection efficiency. Bars indicate means \pm SD from three replicate assays. (C) Potential transcriptional factor binding sites in the cyclin E2 promoter region (-2000---+1) were analyzed via the TRANSFAC 8.3 engine online. (D) Cell lysates from indicated cells were evaluated for OCT2, CDX2, FOXP1, and cyclin E2 protein expression. GAPDH served as loading control.

\section{5. miR-3687 direct targeted 3'-UTR of foxp 1 mRNA and down-regulated its protein expression}

miRNAs play biological roles by modulating target gene expression via binding to the 3 '-UTR of target genes to cause mRNA stability alteration or protein translation suppression [27]. Inhibition of miR-3687 expression increased foxp1 mRNA levels (Figure S2A and S2B), indicating that miR-3687 might reduce foxp1 mRNA stability. Given that the 3'-UTR region of foxp1 mRNA contains potential miR-3687 binding site (Figure 5A), this study next sought to determine whether the effect of miR3687 on FOXP1 protein translation is due to its specific direct binding to the potential binding site in 3'-UTR of foxp1 mRNA. For this, foxp1 mRNA 3'-UTR-driven luciferase reporter (wild-type WT) and foxp1 mRNA 3'-UTR mutant luciferase reporter (MUT) constructs were generated as shown in Figure 5A. The WT and mutant foxp1 3'-UTR luciferase reporter vectors were then transiently transfected into UMUC3(miR-3687i) and T24(miR-3687i) and scramble vector cells with pRL-TK. As seen in Figures 5B and 5C, miR-3687i significantly induced foxp1 3'-UTR WT luciferase reporter activity in both cells, whereas mutation of the miR-3687 binding site at the foxp1 3'-UTR impaired miR-3687imediated increasing of foxp $13^{\prime}$-UTR luciferase reporter activity. This indicates that miR-3687 binds directly to the foxp1 3'-UTR to regulate FOXP1 mRNA stability. 
A

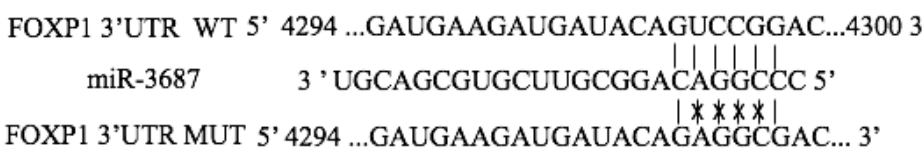

B
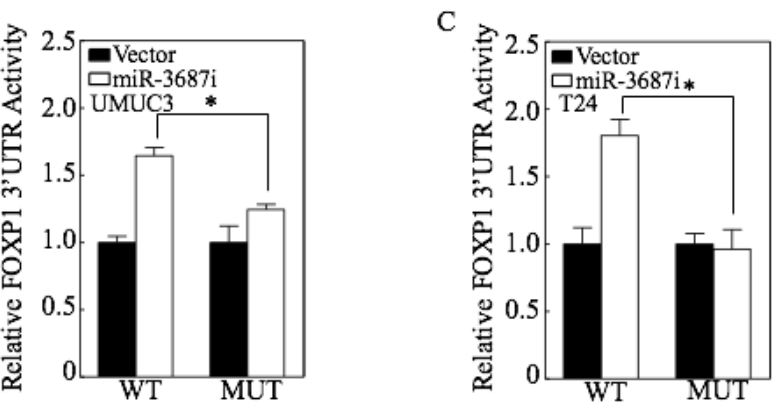

Figure 5. FOXP1 as a target of miR-3687 in human BC cells. (A) Potential miR-3687 targeting sequences of foxp1 mRNA 3'-UTR were analyzed using the TargetScan software. Schematic of construction of foxp1 mRNA 3'-UTR luciferase reporter and its mutants (MUT) were shown. (B) WT and mutant pMIR-FOXP1 3'-UTR reporters were co-transfected with pRL-TK into indicated cells. At $24 \mathrm{~h}$ post-transfection, transfectants were extracted to assess luciferase activity; TK used as internal control. Each bar indicates the mean \pm SD from three assays. *Significant difference $(p<0.05)$.

\subsection{FOXP1 acts as a downstream regulatory gene of miR-3687 to promote its role in promoting $B C$ cell growth}

These above results show that miR-3687 promotes BC cell proliferation and anchorageindependent growth and FOXP1 is a direct target of miR-3687. To determine whether FOXP1 was responsible for the promoting effect of miR-3687 on those two endpoints, FOXP1 expression was knocked down by short hairpin RNA (shRNA) in miR-3687 down-expressing UMUC3 cells. The Western blot analysis showed that shFOXP1 No.1 and No.4 were able to successfully knock down the expression of FOXP1 (Figure 6A). Consistently, knockdown of FOXP1 results in markedly increasing cyclin E2 expression (Figure 6A), cell proliferation and anchorage-independent growth in $\mathrm{BC}$ cells and reversing miR-3687i-inducing $\mathrm{G}_{0} / \mathrm{G}_{1}$ arrest in comparison to scramble nonsense control UMUC3(miR-3687i/Nonsense) cells (Figures 6B-6F). These results suggest that down-expression of FOXP1 reversed the inhibitory effect of miR-3687i in BC cells. Collectively, the data provide evidence that downregulation of FOXP1 is an important event associated with the cancer-promoting activity of miR-3687 in human BC cells. 
A

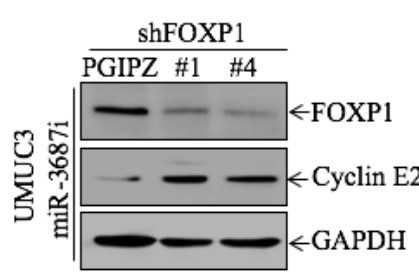

$\mathrm{D}$

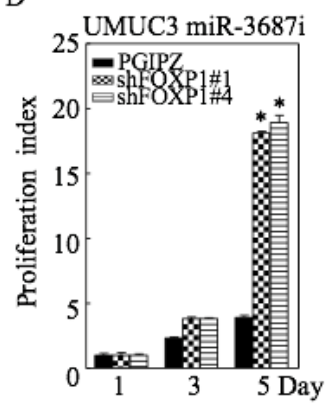

E

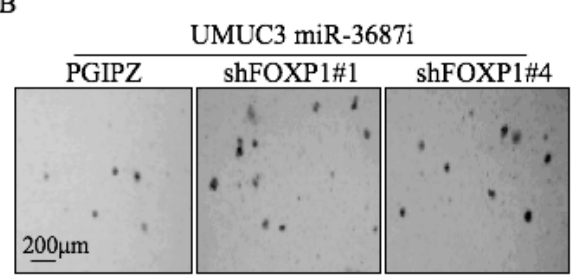

C UMUC3 miR-3687i

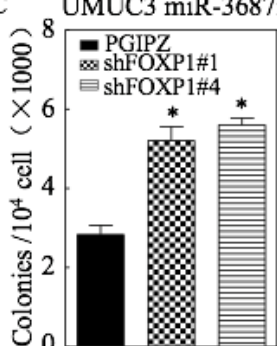

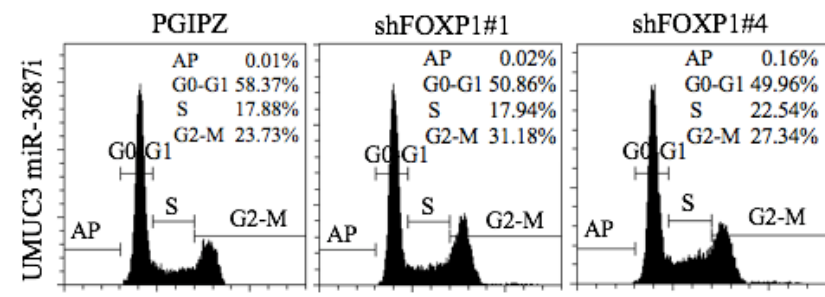

Figure 6. Effects of FOXP1 expression on inhibition of BC cell growth and cell cycle G0/G1 transition. (A) Western blotting was employed to identify the knock-down efficiency of FOXP1 in UMUC3(miR3687i) cells. GAPDH was used as an internal protein loading control. (B and C) Soft agar assay was performed to determine the effect of reduced FOXP1 expression on anchorage-independent growth. (D) Effect of FOXP1 down-expression on monolayer proliferative rates evaluated by ATP assay. Results presented as means \pm SD from triplicates; *significant increase relative to nonsense control cells ( $\mathrm{p}<0.05$ ). (E \& F) Indicated cells were seeded into 6-well plates and cultured to $70-80 \%$ confluence; after synchronization, cells were cultured in complete medium for another $24 \mathrm{~h}$ and then subjected to cell cycle analysis.

\subsection{FOXP1 and cyclin E2 expression correlates in response to miR-3687 inhibition}

Immunohistochemistry (IHC) staining showed that FOXP1 expression was up-regulated in tumor tissues obtained from tumor-bearing mice injected with miR-3687 down-expressed cells as compared with that in the mice injected with corresponding vector cells (Figures 7A \& 7B). In contrast to FOXP1 expression, cyclin E2 expression was decreased in tumor tissues obtained from tumorbearing mice injected within miR-3687 down-expressed cells (Figures 7A-7C). Quantitative analysis of the relative expression of FOXP1 and cyclin E2 in tumor tissues obtained from xenograft nude mice revealed FOXP1, and cyclin E2 expression was negatively correlated in response to miR-3687 downexpression (Figures 7D). Such results are consistent with the in vitro results herein and support the conclusion that miR-3687 acts as a potent oncogene in human BC cells, and that its upregulation may play a critical role in $\mathrm{BC}$ tumorigenesis. 

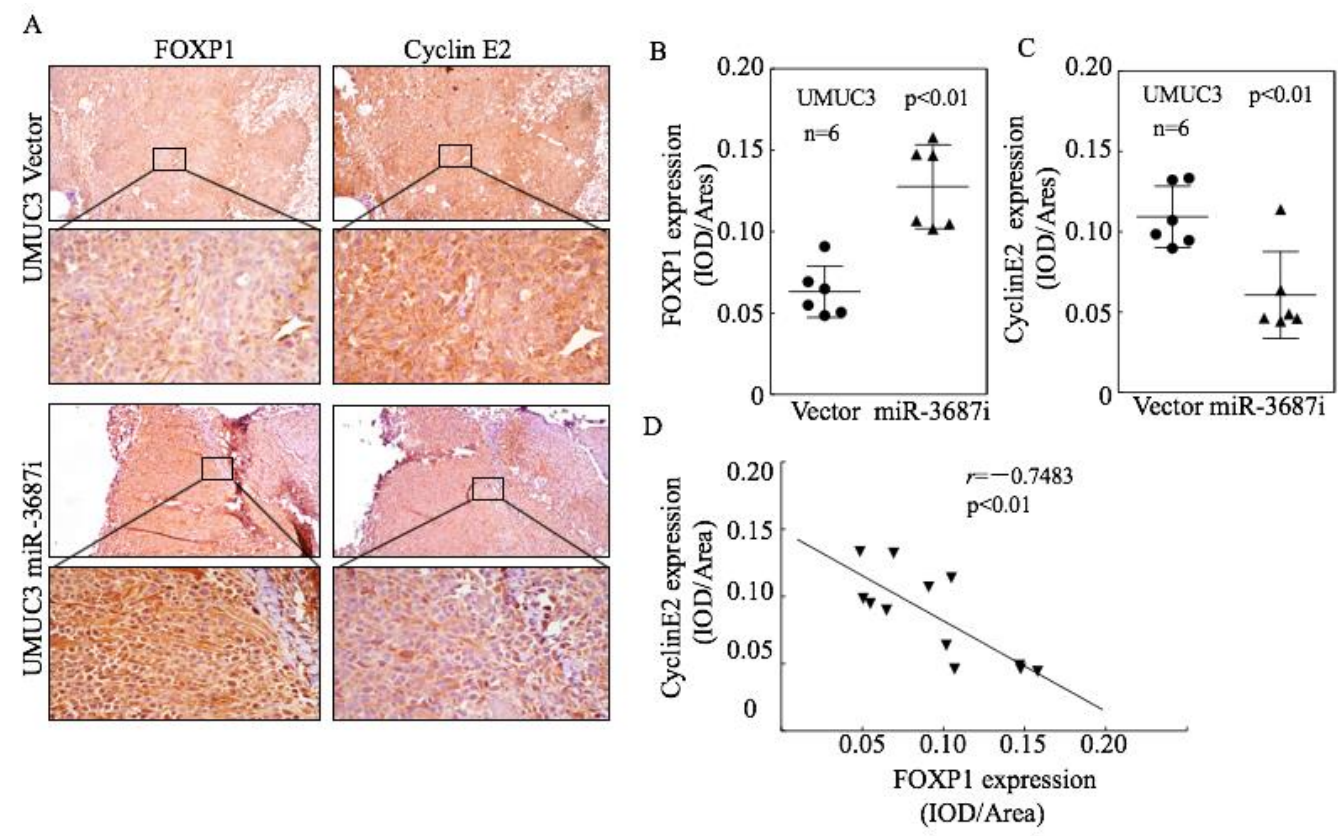

Figure 7. FOXP1 and cyclin E2 were negatively correlated in response to miR-3687 down-expression in the tumor tissues obtained from xenograft nude mice. (A) Representative IHC images showing expression of FOXP1 and cyclin E2 in tumor tissues from nude mice injected with UMUC3(Vector) and UMUC3(miR-3687i) cells. (B and C) The optical density of IHC staining of FOXP1 and cyclin 2E expression was analyzed as described in the section "Materials and Methods." *Significant change in comparison with vector control $(\mathrm{p}<0.01) . \mathrm{IOD} / \mathrm{area}=$ integrated optical density/stained area. (D) Negative correlative expression of FOXP1 with cyclin E2 was analyzed in tumor tissues collected from nude mice injected with indicated cells.

\section{Discussion}

Bladder cancer $(\mathrm{BC})$ is the ninth most common cancer in the world and the 14th leading cause of death. It is a serious international public health problem [28]. According to the American Cancer Society, 79,030 new BC cases were diagnosed in the United States in 2017, with 16,870 death [29]. In China, the age-standardized BC incidence rate is 7.68/100,000, and the standardized mortality rate is 3.03/100,000 [30-32]. At present, the main treatment strategy for early BC is surgery, followed by chemotherapy, radiotherapy, and biological treatment. In patients with advanced $\mathrm{BC}$, it is a prevalent life-threatening malignancy. Therefore, there is an urgent need to develop new diagnostic and therapeutic targets so that BC can be caught earlier [33]. Since tumor development in BC involves genetic alterations, epigenetic changes, and environmental factors [34], it is also critical to clarify the molecular mechanisms underlying development/progression of BC so as to improve diagnostic accuracy and clinical treatment.

MicroRNAs (miRNA or miR) are small non-coding single-stranded RNA of 19-25 nucleotides (nt) in length and act as regulators of gene expression at the post-transcriptional level [35]. miRNAs may also act as tumor suppressors or as oncogenes [36]. Recent literature suggests that miRNAs play an essential role in various biological processes related to cancer, including cell differentiation, proliferation, tumorigenesis, angiogenesis, invasion, and metastasis [37,38]. Previous reports have shown that the expression of miR-3687 is elevated in cancer, including renal cell carcinoma (RCC) and upper urinary tract urothelial carcinoma (UT-UC) [39]. This study suggests that dysregulation of miR-3687 may be a common manifestation of renal cancer development/progression. miR-3687 is also elevated in other types of cancers. For example, miR-3687 is elevated in conjunctival melanoma and associated with a higher risk of local recurrence of conjunctival melanoma [40]. Compared to in control cells, miR-3687 is up-regulated in Het-1A, immortalized non-tumorigenic esophageal epithelial cells treated with $20 \%$ cigarette smoke [41]. On the other hand, miR-3687 is down-regulated 
in breast cancer and colon cancer cell lines [42]. Therefore, the role directly attributed to miR-3687 in enhancing cancer risk (in specific organ systems) remains controversial. However, in the study here, the TCGA database and fresh clinic human BC paired tissues collected from this study were used to assess miR-3687 expression in BC. miR-3687 levels are elevated in BC and promote BC cell growth both in vitro and in vivo by up-regulating cyclin E2 expression.

Cyclin E2 is up-regulated in many tumor types and appears to induce G0/G1 cell cycle arrest in human BC cells. Cyclin E2 is regulated by a variety of mechanisms at the transcriptional/posttranscriptional levels. Our data indicate that inhibition of miR-3687 expression results in downregulation of cyclin E2 mRNA levels and cyclin E2 promoter activity, indicating that cyclin E2 is regulated at a transcriptional level. TRANSFAC® transcription factor binding site software showed the cyclin E2 promoter region contains putative DNA binding sites for OCT2, CDX2, and FOXP1. Our data indicate that expression of FOXP1 was up-regulated in UMUC3(miR-3687i) and T24(miR-3687i) compared to scramble nonsense control cells, while that there is no significant change in OCT2 or CDX2. Further, we show that knockdown of FOXP1 remarkably increase cyclin E2 expression, BC cell growth as well as promoting G0/G1 cell cycle transition in miR-3687-inhibited BC cells. Therefore, we draw the conclusion that FOXP1 is a downstream transcriptional regulator of miR-3687 that negatively regulates cyclin E2, which in turn binds the cyclin E2 promoter and inhibits cyclin E2 transcription, resulting in inhibition of the G0/G1 cell cycle transition, and reduction in BC cell proliferation and tumor growth.

Forkhead box (FOX) proteins constitute a large class of transcription factors with multiple functions, from development and organogenesis to regulation of metabolic and immune functions [43]. The Fox transcription factor is characterized by a 100 amino acid wing helix/forkhead DNA binding domain. In addition, the Fox protein subfamily, including FOXP1-P4, contains a zinc finger domain and a leucine zipper motif and can act as a transcriptional repressor by forming homo- or heterodimers with other family members. The function of FOXP1 has been widely studied in blood, lung, heart and immune cells [44-46]. Other studies have shown FOXP1 can act as an oncogene in diffuse large B-cell lymphoma [47], mucosal-associated lymphoid tissue lymphoma [48] or act as a tumor suppressor in gastrointestinal [49], lung cancer [50], genitourinary, and breast cancer [51]. In the present study, FOXP1 is found to be responsible for the attenuation of cyclin E2 expression by miR-3687i at the transcriptional level. Since FOXP1 can transcriptionally inhibit the transcription of cyclin E2, indicating that FOXP1 acts as a tumor suppressor in BC cells, which has not been reported in previous studies.

In summary, the current study showed for the first time that miR-3687 was up-regulated in BC tissue and cell lines. Functional studies revealed that miR-3687 promotes BC cell growth in vitro and tumor formation in vivo, and down-regulates FOXP1 expression (by targeting it's 3'-UTR). Subsequent reduction of FOXP1 promotes the transcription of cyclin E2 by decreasing its binding to the cyclin E2 promoter. Eventually, increased levels of cyclin E2 promote BC cell proliferation through promoting the G0/G1 transition. Taken together, these results indicate that miR-3687 is a critical cancer-promoting molecule in BC, and that miR-3687 and its downstream effectors may serve as potential targets for early diagnosis and/or target during the treatment of $\mathrm{BC}$ patients.

\section{Materials and Methods}

\subsection{Reagents, antibodies, and plasmids}

Sponge inhibitor explicitly targeting miR-3687 and its control vector plasmids were purchased from GenePharma (\#C5619, Shanghai, China). The human foxp1 3'-untranslated region (3'-UTR) was cloned into the pMIR-Report luciferase vector through the KpnI and HindIII sites. The FOXP1 3'-UTR point mutation was amplified from the wild-type (WT) template by overlap PCR using the primers (F) 5'-ATT TAT CTA TCT GTC CTA ACC ATT TCT CTC AAA A-3' and (R) 5'-TTT TGA GAG AAA TGG TTA GGA CAG ATA GAT AAT-3'. The dual luciferase assay kit was bought from Promega (Madison, WI, USA), while TRIzol and a SuperScript ${ }^{\mathrm{TM}}$ First-Strand Synthesis system were purchased 
from Invitrogen (Grand Island, NY, USA). shRNA specifically targeting human FOXP1 were bought from Open Biosystems (GE Healthcare, Pittsburgh, PA, USA). The cyclin E2 expression plasmid was bought from Obio Technology (Shanghai, China). The cyclin E2 promoter-driven luciferase reporter was obtained from Addgene (Cambridge, MA). Specific antibodies against P21 (sc-397), CDK2 (sc70829), CDK4 (sc-260), CDK6 (sc-177), cyclinD1 (sc-20044) were all bought from Santa Cruz Biotechnology (Santa Cruz, CA, USA). Specific antibodies against FOXP1 (CST\#4402), cyclin E2 (CST\#4132), GAPDH (CST\#5174) were all purchased from Cell Signaling Technology (Boston, MA, USA). Specific antibodies against CDX2 (GTX\#32513) was bought from genetex (Irvine, CA, USA) and OCT2 (\#18996-1-AP) was bought from Proteintech (Chicago, IL, USA).

\subsection{Cell culture and transfection}

The human BC cell lines T24, T24T, UMUC3, J82, and TCCSUP, and the normal urinary epithelial cell line UROtsa were used in this study. All BC cell lines were subjected to DNA tests and authenticated before use. UROtsa was generously provided by Dr. Scott H. Garrent (University of North Dakota, Grand Forks, ND) and used in our previous studies (Jin H, [24,52]). UMUC3 cells were maintained at $37^{\circ} \mathrm{C}$ in a 5\% CO2 incubator in DMEM (\#11995-065, Gibco, Grand Island, NY, USA) supplemented with 10\% fetal bovine serum (FBS; \#10437-028, Gibco). T24 and T24T cells were cultured with a 1:1 mixture of DMEM/Ham's F12 medium (\#10565-018, Gibco) supplemented with 5\% FBS. UROtsa cells were cultured in RPMI 1640 (\#11875-093, Gibco) supplemented with 10\% FBS. TCCSUP and J82 cells were cultured with MEM (\#11095-080, Gibco) supplemented with 10\% FBS. Stable transfections were performed with constructs using PolyJet ${ }^{\mathrm{TM}}$ DNA In Vitro Transfection Reagent (SignaGen Laboratories, Gaithersburg, MD, USA) according to manufacturer's instructions. Stable transfectants were selected with puromycin $(0.2-0.3 \mu \mathrm{g} / \mathrm{ml})$ or hygromycin B $(200-400 \mu \mathrm{g} / \mathrm{ml})$ for 3-4 wk, according to the different antibiotic resistance plasmids transfected.

\subsection{Lentivirus packaging and infection}

Stable FOXP1 knockdown cells were established by lentivirus infection as described in our published studies [53]. Briefly, 293T cells were seeded into 6-well plates until reaching 60-70\% confluence. The transfection complex consisted of $2.0 \mu \mathrm{g}$ DNA plasmid and two packaging vectors (1.2 $\mu \mathrm{g}$ pMD2.G and 1.2 $\mu \mathrm{g}$ psPAX2) diluted in $100 \mu \mathrm{l}$ serum-free DMEM, and $4.0 \mu$ l PolyJet ${ }^{\mathrm{TM}}$ DNA (SignaGen Laboratories, Rockville, MD, USA) diluted in $100 \mu$ serum-free DMEM. The diluted PolyJet $^{\mathrm{TM}}$ reagent was immediately added to the diluted DNA solution, and the mixture was shaken and then incubated for $15 \mathrm{~min}$ at room temperature to form a transfection complex. The latter, in turn, was added to the $293 \mathrm{~T}$ cells, and viral supernatant fractions produced at $48 \mathrm{~h}$ were isolated, purified by centrifugation at $2500 \mathrm{rpm}$ for $30 \mathrm{~min}$, and filtered through a $0.45 \mu \mathrm{m}$ pore membrane. Viral supernatant fractions were used to transfect target cells at a ratio of $0.1 \mathrm{ml}: 1 \mathrm{ml}$ (viral supernatant fraction: medium). Cells were cultured in 6-well plates, and after $48 \mathrm{~h}$, the medium was replaced with complete fresh medium containing $3.0 \mu \mathrm{g}$ puromycin $/ \mathrm{ml}$. Similar experiments were performed with all cells until the control cells (without infections) completely died (2-3 days) in the puromycincontaining medium.

\subsection{Anchorage-independent growth}

The potential inhibitory effect of miR-3687i on anchorage-independent growth of human BC cells was determined in soft agar assay as described in our published studies [54]. In brief, 104 UMUC3(miR-3687i) or T24(miR-3687i) stable transfectants and control vector transfectants were exposed to Basal Medium Eagle (BME) containing 0.33\% agar and seeded on the bottom layer of $0.5 \%$ agar in $10 \%$ FBS-BME in each well of 6 -well plates. The cultures were then maintained at $37^{\circ} \mathrm{C}$ in a $5 \%$ CO2 incubator for 3-4 wk. Wells containing cell colonies with $>32$ cells were then scored. Colonies were observed and counted using a DMi1 microscope (Leica, Frankfurt am Main, Germany). The results were presented as [mean \pm SD] colonies $/ 10,000$ seeded cells.

\subsection{Monolayer cell proliferation}


Confluent monolayers of UMUC3 and T24 cells were trypsinized with $0.25 \%$ trypsin (\#25200072 , Gibco), and then $0.4 \times 103$ viable cells suspended in $200 \mu \mathrm{l}$ complete medium was added to each well of 96 -well plates. After adherence $\left(6 \mathrm{~h}\right.$ at $\left.37^{\circ} \mathrm{C}\right)$, cells were synchronized by replacing with culture medium containing only $0.1 \%$ FBS for $24 \mathrm{~h}$. Thereafter, the cells were cultured in complete medium for the indicated days. At each point, the cell proliferation index was determined using a Cell TiterGlo Luminescent Cell Viability Assay kit (Promega). Each time, luminescence was measured with a Centro LB 960 luminometer (Berthold Technologies, Berthold, Germany).

\subsection{Western blot}

Cells were pelleted and then lysed on ice in a "boiling buffer" containing 1\% SDS, $1 \mathrm{mM}$ $\mathrm{Na} 3 V O 4,10 \mathrm{mM}$ Tris- $\mathrm{HCl}$ ( $\mathrm{pH} 7.4$ ). The materials were heated at $100^{\circ} \mathrm{C}$ for $5-10 \mathrm{~min}$, and then ultrasonicated to break all nucleic acids. Protein concentration in the final suspension was determined using the BCA Protein Assay kit (ThermoScientific, Pittsburgh, PA, USA). Samples of each cell extract (40 $\mathrm{\mu g} /$ lane) were then subjected to SDS-PAGE, and the resolved proteins then electrotransferred to polyvinylidene fluoride (PVDF) membranes (BioRad, Hercules, CA, USA). After blocking with 5\% non-fat milk in TBST, each dedicated membrane was incubated at $4^{\circ} \mathrm{C}$ overnight in a solution of $5 \%$ BSA containing a given primary rabbit antibody CDK6 [1:1000], CDK4 [1:1000], CyclinE2 [1:1000], FOXP1 [1:1000], OCT2 [1:800], CDX2 [1:800], or p21 [1:800]. To evaluate Cyclin D1 and CDK2 a mouse antibody was employed [1:1000]. All blots were probed simultaneously with rabbit anti-GAPDH [1:3000] or -Tubulin [1:3000]. The membranes were then gently rinsed with TBST and then incubated for $3 \mathrm{~h}$ at $4^{\circ} \mathrm{C}$ in a solution of containing $5 \%$ non-fat milk alkaline phosphatase (AP)-conjugated secondary antibody (anti-mouse or anti-rabbit IgG, 1:2000 dilution). Signals were then detected using an ECF Western-blotting system and a Typhoon FLA 7000 imager (GE Healthcare).

\subsection{Quantitative RT-PCR}

Total miRNA were extracted using a miRNeasy Mini Kit (Qiagen, Valencia, CA) and total RNA $(1.0 \mu \mathrm{g})$ was then used for reverse transcription following manufacturer instructions. miRNA expression was determined using a Q6 real-time PCR system (Applied Biosystems, Carlsbad, CA) and a miScript PCR Kit (Qiagen). The 5'-TAGTAGACCGTATAGCGTACG-3' primer for miR-3687 was synthesized by Sunny Biotechnology (Shanghai, China). U6 was used as an internal loading control. Cycle threshold (CT) values were determined, and the relative expression of miRNA calculated from the values of $2-\Delta \Delta \mathrm{CT}$.

\subsection{Dual-luciferase reporter assay}

UMUC3(Vector), UMUC3(miR-3687i), T24(Vector), and T24(miR-3687i) cells were transiently co-transfected for $24 \mathrm{~h}$ with a foxp1 3'-UTR WT or FOXP1 3'-UTR mutant luciferase reporter and pRLTK. The transfectants were then washed with PBS and extracted with passive lysis buffer at room temperature for $15 \mathrm{~min}$ to ensure complete lysis of the cells. Cell lysates were transferred to a 96-well plate, and Luciferase Assay Reagent II (as part of Dual-Luciferase ${ }^{\circledR}$ Reporter Assay System [Promega]) was added. After $15 \mathrm{~min}$ at room temperature, kit-provided Stop\&Glo Reagent was added to the wells, and pRL-TK activity was assessed using a Centro LB 960 luminometer (Berthold $\mathrm{GmbH}$, Bad Wildbad, Germany). The WT and mutant cyclin E2 promoter-driven luciferase reporter in the FOXP1 binding site were the same as the foxp1 3'-UTR.

\subsection{Xenograft model in nude mice in vivo}

Female BALB/c athymic nude mice (3-4 wk old) were purchased from the Silaike Experimental Animal Company (Shanghai). All mice were housed in specific pathogen-free facilities at the Animal Institute of Wenzhou Medical University that were maintained at $26^{\circ} \mathrm{C}$ with $40 \%$ relative humidity and a 12-h light/dark cycle. All mice had ad libitum access to standard rodent chow and filtered tap water. After a 2-wk acclimatization, mice were randomly allocated into four groups and subcutaneously injected in the right side (lower back) with $0.1 \mathrm{ml}$ T24(miR-3687i) and T24(Vector) or 
UMUC3(miR-3687) and UMUC3(Vector) cells (in each case, $2.0 \times 106$ cells suspended in $100 \mu 1$ PBS). After $4-5 \mathrm{wk}$, the mice were sacrificed, and any tumor present was surgically removed, imaged, and weighed. One-third of each total tumor was then fixed in $4 \%$ paraformaldehyde for later used in immunohistochemical analyses (IHC), one third was frozen at $-80^{\circ} \mathrm{C}$, and the remaining third was used to extract RNA (if necessary). All animal studies were performed using protocols approved by the Laboratory Animal Ethics Committee of Wenzhou Medical University (ID Number: wydw20170073; Approval Date: March 4, 2017).

\subsection{Immunohistochemistry (IHC)}

Tumor tissues from the mice were formalin-fixed and paraffin-embedded. For IHC staining, antibodies against cyclin E2 (1:200 dilution; \#ab109016, Abcam, Cambridge, UK) and FOXP1 (1:200 dilution, \#sc-397, Santa Cruz Biotechnology, CA, USA) were used. Staining was done using a commercial kit (Boster Bio-Engineering Company, Wuhan, China) according to manufacturer instructions. Resultant immunostained sample images were captured using a DS-Ri2 Eclipse Ni microsystem (Nikon, Tokyo, Japan). Protein expression levels were analyzed by calculating integrated optical density/stained area (IOD/area) using Image-Pro Plus (v.6.0, Media Cybernetics, Bethesda, MD, USA).

\subsection{Clinical specimens}

The presented study was approved by the Ethetics Committee of Wenzhou Medical University (ID Number: No.KT2016-9; Approval Date: March 10, 2016). To provide a background for the actual expression of miRNA-3687 in bladder cancer, human BC tissue samples and corresponding adjacent normal tissues were isolated from patients at the First Affiliated Hospital of Wenzhou Medical University (Zhejiang, China). All patients provided informed consent to having the biosamples collected for study. In total, 19 pairs of BC/normal tissues were collected and detailed information was described previously(23). Each sample was snap frozen in liquid nitrogen at the time of surgery; subsequently, RNA was extracted, and cDNA was synthesized (using standard protocols) and stored at $-80^{\circ} \mathrm{C}$ until analysis.

\subsection{Statistical analysis}

A Student's t-test was used to determine the significance between any two groups of interest. Results are expressed as mean ๑ SD from at least three independent experiments. A p-value of $<0.05$ was accepted as a significant difference between the specified two groups that were compared. All data were analyzed using GraphPad software (La Jolla, CA, USA).

Supplementary Materials: Figure S1: TCGA database analysis of the expression of miR-3687 in human bladder cancer tissues and adjacent tissues $(\mathrm{n}=19)$., Figure S2: miR-3687 downregulated foxp1 mRNA stability.

Author Contributions: Q.X., C.C., H.H. and C.H. conceptualized the idea and designed the experiments. H.L., H.Y., J.X., D.R., H.Z. and J.H. conducted the experiments and analyzed the data. Q.X., H.H., and C.H., wrote the paper. All authors read and approved the manuscript.

Funding: This work was partially supported by the Natural Science Foundation of China (NSFC81601849, NSFC81702530, NSFC81773391 and NSFC81872587); Wenzhou Science and Technology Bureau (Y20170028 and Y20160075); Key Discipline of Zhejiang Province in Medical Technology (First Class, Category A); the Key Project of Science and Technology Innovation Team of Zhejiang Province (2013TD10); and the grants of NIH/NCI CA165980, CA217923, CA177665, and NIH/NIEHS ES000260.

Acknowledgments: The results published here are in whole based on data generated by the TCGA Research Network: (http://cancergenome.nih.gov/). We also thank the participants, specimen donors and research groups who developed the TCGA bladder cancer dataset resource for their contributions in database construction.

Conflicts of Interest: The authors declare no conflicts of interest. 


\section{References}

1. Lauper, N.; Beck, A.R.; Cariou, S.; Richman, L.; Hofmann, K.; Reith, W.; Slingerland, J.M.; Amati, B. Cyclin E2: a novel CDK2 partner in the late G1 and S phases of the mammalian cell cycle. Oncogene 1998, 17, 2637-2643, doi:10.1038/sj.onc.1202477.

2. Zariwala, M.; Liu, J.; Xiong, Y. Cyclin E2, a novel human G1 cyclin and activating partner of CDK2 and CDK3, is induced by viral oncoproteins. Oncogene 1998, 17, 2787, doi:10.1038/sj.onc.1202505.

3. Caldon, C.E.; Musgrove, E.A. Distinct and redundant functions of cyclin E1 and cyclin E2 in development and cancer. Cell Div 2010, 5, 2, doi:10.1186/1747-1028-5-2.

4. Hwang, H.C.; Clurman, B.E. Cyclin E in normal and neoplastic cell cycles. Oncogene 2005, 24, 2776-2786, doi:10.1038/sj.onc.1208613.

5. Santamaria, D.; Ortega, S. Cyclins and CDKS in development and cancer: lessons from genetically modified mice. Front Biosci 2006, 11, 1164-1188, doi:10.2741/1871

6. Iida, H.; Towatari, M.; Tanimoto, M.; Morishita, Y.; Kodera, Y.; Saito, H. Overexpression of cyclin E in acute myelogenous leukemia. Blood 1997, 90, 3707-3713.

7. Desmedt, C.; Ouriaghli, F.E.; Durbecq, V.; Soree, A.; Colozza, M.A.; Azambuja, E.; Paesmans, M.; Larsimont, D.; Buyse, M.; Harris, A., et al. Impact of cyclins E, neutrophil elastase and proteinase 3 expression levels on clinical outcome in primary breast cancer patients. Int J Cancer 2006, 119, 25392545, doi:10.1002/ijc.22149.

8. $\quad$ Sieuwerts, A.M.; Look, M.P.; Meijer-van Gelder, M.E.; Timmermans, M.; Trapman, A.M.; Garcia, R.R.; Arnold, M.; Goedheer, A.J.; de Weerd, V.; Portengen, H. Which cyclin E prevails as prognostic marker for breast cancer? Results from a retrospective study involving 635 lymph node-negative breast cancer patients. Clinical Cancer Research 2006, 12, 3319-3328.

9. Pornchai, O.; Rusch, V.; Talbot, S.G.; Sarkaria, I.; Viale, A.; Socci, N.; Ngai, I.; Rao, P.; Singh, B. Casein kinase II alpha subunit and C1-inhibitor are independent predictors of outcome in patients with squamous cell carcinoma of the lung. Clinical Cancer Research 2004, 10, 5792-5803.

10. Shridhar, V.; Lee, J.; Pandita, A.; Iturria, S.; Avula, R.; Staub, J.; Morrissey, M.; Calhoun, E.; Sen, A.; Kalli, K., et al. Genetic analysis of early- versus late-stage ovarian tumors. Cancer Res 2001, 61, 58955904.

11. Chen, H.C.; Chen, G.H.; Chen, Y.H.; Liao, W.L.; Liu, C.Y.; Chang, K.P.; Chang, Y.S.; Chen, S.J. MicroRNA deregulation and pathway alterations in nasopharyngeal carcinoma. Br J Cancer 2009, 100, 1002-1011, doi:10.1038/sj.bjc.6604948.

12. Gudas, J.M.; Payton, M.; Thukral, S.; Chen, E.; Bass, M.; Robinson, M.O.; Coats, S. Cyclin E2, a novel G1 cyclin that binds Cdk2 and is aberrantly expressed in human cancers. Molecular and cellular biology 1999, 19, 612-622.

13. Deng, J.; He, M.; Chen, L.; Chen, C.; Zheng, J.; Cai, Z. The loss of miR-26a-mediated post-transcriptional regulation of cyclin E2 in pancreatic cancer cell proliferation and decreased patient survival. PLoS One 2013, 8, e76450, doi:10.1371/journal.pone.0076450.

14. Müller-Tidow, C.; Metzger, R.; Kügler, K.; Diederichs, S.; Idos, G.; Thomas, M.; Dockhorn-Dworniczak, B.; Schneider, P.M.; Koeffler, H.P.; Berdel, W.E. Cyclin E is the only cyclin-dependent kinase 2associated cyclin that predicts metastasis and survival in early stage non-small cell lung cancer. Cancer research 2001, 61, 647-653. 
15. Wu, L.; Timmers, C.; Maiti, B.; Saavedra, H.I.; Sang, L.; Chong, G.T.; Nuckolls, F.; Giangrande, P.; Wright, F.A.; Field, S.J., et al. The E2F1-3 transcription factors are essential for cellular proliferation. Nature 2001, 414, 457-462, doi:10.1038/35106593.

16. Lee, Y.; Kim, M.; Han, J.; Yeom, K.H.; Lee, S.; Baek, S.H.; Kim, V.N. MicroRNA genes are transcribed by RNA polymerase II. EMBO J 2004, 23, 4051-4060, doi:10.1038/sj.emboj.7600385.

17. He, L.; Thomson, J.M.; Hemann, M.T.; Hernando-Monge, E.; Mu, D.; Goodson, S.; Powers, S.; CordonCardo, C.; Lowe, S.W.; Hannon, G.J. A microRNA polycistron as a potential human oncogene. nature 2005, 435, 828 .

18. Zhang, B.; Pan, X.; Cobb, G.P.; Anderson, T.A. microRNAs as oncogenes and tumor suppressors. Developmental biology 2007, 302, 1-12.

19. He, L.; He, X.; Lim, L.P.; de Stanchina, E.; Xuan, Z.; Liang, Y.; Xue, W.; Zender, L.; Magnus, J.; Ridzon, D., et al. A microRNA component of the p53 tumour suppressor network. Nature 2007, 447, 1130-1134, doi:10.1038/nature05939.

20. Welch, C.; Chen, Y.; Stallings, R. MicroRNA-34a functions as a potential tumor suppressor by inducing apoptosis in neuroblastoma cells. Oncogene 2007, 26, 5017.

21. Yoshino, H.; Seki, N.; Itesako, T.; Chiyomaru, T.; Nakagawa, M.; Enokida, H. Aberrant expression of microRNAs in bladder cancer. Nat Rev Urol 2013, 10, 396-404, doi:10.1038/nrurol.2013.113.

22. Lu, J.; Getz, G.; Miska, E.A.; Alvarez-Saavedra, E.; Lamb, J.; Peck, D.; Sweet-Cordero, A.; Ebert, B.L.; Mak, R.H.; Ferrando, A.A., et al. MicroRNA expression profiles classify human cancers. Nature 2005, 435, 834-838, doi:10.1038/nature03702.

23. Lin, S.; Gregory, R.I. MicroRNA biogenesis pathways in cancer. Nat Rev Cancer 2015, 15, 321-333, doi:10.1038/nrc3932.

24. Jin, H.; Sun, W.; Zhang, Y.; Yan, H.; Liufu, H.; Wang, S.; Chen, C.; Gu, J.; Hua, X.; Zhou, L., et al. MicroRNA-411 Downregulation Enhances Tumor Growth by Upregulating MLLT11 Expression in Human Bladder Cancer. Mol Ther Nucleic Acids 2018, 11, 312-322, doi:10.1016/j.omtn.2018.03.003.

25. Koon, H.B.; Ippolito, G.C.; Banham, A.H.; Tucker, P.W. FOXP1: a potential therapeutic target in cancer. Expert Opin Ther Targets 2007, 11, 955-965, doi:10.1517/14728222.11.7.955.

26. Bacon, C.; Rappold, G.A. The distinct and overlapping phenotypic spectra of FOXP1 and FOXP2 in cognitive disorders. Hum Genet 2012, 131, 1687-1698, doi:10.1007/s00439-012-1193-z.

27. Lai, E.C. Micro RNAs are complementary to $3^{\prime}$ UTR sequence motifs that mediate negative posttranscriptional regulation. Nature genetics 2002, 30, 363.

28. Mahdavifar, N.; Ghoncheh, M.; Pakzad, R.; Momenimovahed, Z.; Salehiniya, H. Epidemiology, incidence and mortality of bladder cancer and their relationship with the development index in the world. Asian Pac J Cancer Prev 2016, 17, 381-386.

29. Siegiel, R.; Miller, K.; Jemal, A. Cancer Statistics, 2017. CA Cancer J Clin 2017, 67, 7-30.

30. Chen, W.; Zheng, R.; Baade, P.D.; Zhang, S.; Zeng, H.; Bray, F.; Jemal, A.; Yu, X.Q.; He, J. Cancer statistics in China, 2015. CA Cancer J Clin 2016, 66, 115-132, doi:10.3322/caac.21338.

31. Chen, W.; Zheng, R.; Zeng, H.; Zhang, S.; He, J. Annual report on status of cancer in China, 2011. Chin J Cancer Res 2015, 27, 2-12, doi:10.3978/j.issn.1000-9604.2015.01.06.

32. Chen, W.; Zheng, R.; Zhang, S.; Zhao, P.; Zeng, H.; Zou, X.; He, J. Annual report on status of cancer in China, 2010. Chin J Cancer Res 2014, 26, 48-58, doi:10.3978/j.issn.1000-9604.2014.01.08.

33. Ploeg, M.; Aben, K.K.; Kiemeney, L.A. The present and future burden of urinary bladder cancer in the world. World J Urol 2009, 27, 289-293, doi:10.1007/s00345-009-0383-3. 
34. Luke, C.; Tracey, E.; Stapleton, A.; Roder, D. Exploring contrary trends in bladder cancer incidence, mortality and survival: implications for research and cancer control. Intern Med J 2010, 40, 357-362, doi:10.1111/j.1445-5994.2009.01980.x.

35. Ambros, V. The functions of animal microRNAs. Nature 2004, 431, 350-355, doi:10.1038/nature02871.

36. Shenouda, S.K.; Alahari, S.K. MicroRNA function in cancer: oncogene or a tumor suppressor? Cancer Metastasis Rev 2009, 28, 369-378, doi:10.1007/s10555-009-9188-5.

37. Winter, J.; Diederichs, S. MicroRNA biogenesis and cancer. In MicroRNA and Cancer, Springer: 2011; pp. 3-22.

38. Calin, G.A.; Croce, C.M. MicroRNA signatures in human cancers. Nat Rev Cancer 2006, 6, 857-866, doi:10.1038/nrc1997.

39. Zaravinos, A.; Lambrou, G.I.; Mourmouras, N.; Katafygiotis, P.; Papagregoriou, G.; Giannikou, K.; Delakas, D.; Deltas, C. New miRNA profiles accurately distinguish renal cell carcinomas and upper tract urothelial carcinomas from the normal kidney. PLoS One 2014, 9, e91646, doi:10.1371/journal.pone.0091646.

40. Larsen, A.C.; Mikkelsen, L.H.; Borup, R.; Kiss, K.; Toft, P.B.; von Buchwald, C.; Coupland, S.E.; Prause, J.U.; Heegaard, S. MicroRNA Expression Profile in Conjunctival Melanoma. Invest Ophthalmol Vis Sci 2016, 57, 4205-4212, doi:10.1167/iovs.16-19862.

41. Gong, J.; Chu, Y.; Xu, M.; Huo, J.; Lv, L. Esophageal squamous cell carcinoma cell proliferation induced by exposure to low concentration of cigarette smoke extract is mediated via targeting miR-101-3p/COX2 pathway. Oncology reports 2016, 35, 463-471.

42. Reza, A.M.M.T.; Choi, Y.-J.; Yuan, Y.-G.; Das, J.; Yasuda, H.; Kim, J.-H. MicroRNA-7641 is a regulator of ribosomal proteins and a promising targeting factor to improve the efficacy of cancer therapy. Scientific reports 2017, 7, 8365 .

43. Carlsson, P.; Mahlapuu, M. Forkhead transcription factors: key players in development and metabolism. Dev Biol 2002, 250, 1-23.

44. Shu, W.; Yang, H.; Zhang, L.; Lu, M.M.; Morrisey, E.E. Characterization of a new subfamily of wingedhelix/forkhead (Fox) genes that are expressed in the lung and act as transcriptional repressors. Journal of Biological Chemistry 2001, 276, 27488-27497.

45. Wang, B.; Weidenfeld, J.; Lu, M.M.; Maika, S.; Kuziel, W.A.; Morrisey, E.E.; Tucker, P.W. Foxp1 regulates cardiac outflow tract, endocardial cushion morphogenesis and myocyte proliferation and maturation. Development 2004, 131, 4477-4487, doi:10.1242/dev.01287.

46. Hu, H.; Wang, B.; Borde, M.; Nardone, J.; Maika, S.; Allred, L.; Tucker, P.W.; Rao, A. Foxp1 is an essential transcriptional regulator of B cell development. Nature immunology 2006, 7, 819.

47. Banham, A.H.; Connors, J.M.; Brown, P.J.; Cordell, J.L.; Ott, G.; Sreenivasan, G.; Farinha, P.; Horsman, D.E.; Gascoyne, R.D. Expression of the FOXP1 transcription factor is strongly associated with inferior survival in patients with diffuse large B-cell lymphoma. Clinical Cancer Research 2005, 11, 1065-1072.

48. Remstein, E.D.; Dogan, A.; Einerson, R.R.; Paternoster, S.F.; Fink, S.R.; Law, M.; Dewald, G.W.; Kurtin, P.J. The incidence and anatomic site specificity of chromosomal translocations in primary extranodal marginal zone B-cell lymphoma of mucosa-associated lymphoid tissue (MALT lymphoma) in North America. Am J Surg Pathol 2006, 30, 1546-1553, doi:10.1097/01.pas.0000213275.60962.2a.

49. Banham, A.H.; Beasley, N.; Campo, E.; Fernandez, P.L.; Fidler, C.; Gatter, K.; Jones, M.; Mason, D.Y.; Prime, J.E.; Trougouboff, P. The FOXP1 winged helix transcription factor is a novel candidate tumor suppressor gene on chromosome 3p. Cancer research 2001, 61, 8820-8829. 
50. Shimizu, K.; Kato, A.; Hinotsume, D.; Shigemura, M.; Hanaoka, M.; Shimoichi, Y.; Honoki, K.; Tsujiuchi, T. Reduced expressions of Foxp1 and Rassfla genes in lung adenocarcinomas induced by Nnitrosobis(2-hydroxypropyl)amine in rats. Cancer Lett 2006, 236, 186-190, doi:10.1016/j.canlet.2005.05.031.

51. Fox, S.B.; Brown, P.; Han, C.; Ashe, S.; Leek, R.D.; Harris, A.L.; Banham, A.H. Expression of the forkhead transcription factor FOXP1 is associated with estrogen receptor $\alpha$ and improved survival in primary human breast carcinomas. Clinical Cancer Research 2004, 10, 3521-3527.

52. Huang, H.; Jin, H.; Zhao, H.; Wang, J.; Li, X.; Yan, H.; Wang, S.; Guo, X.; Xue, L.; Li, J. RhoGDI $\beta$ promotes Sp1/MMP-2 expression and bladder cancer invasion through perturbing miR-200c-targeted JNK2 protein translation. Molecular oncology 2017, 11, 1579-1594.

53. Huang, H.; Pan, X.; Jin, H.; Li, Y.; Zhang, L.; Yang, C.; Liu, P.; Liu, Y.; Chen, L.; Li, J., et al. PHLPP2 Downregulation Contributes to Lung Carcinogenesis Following B[a]P/B[a]PDE Exposure. Clin Cancer Res 2015, 21, 3783-3793, doi:10.1158/1078-0432.CCR-14-2829.

54. Xie, Q.; Guo, X.; Gu, J.; Zhang, L.; Jin, H.; Huang, H.; Li, J.; Huang, C. p85alpha promotes nucleolin transcription and subsequently enhances EGFR mRNA stability and EGF-induced malignant cellular transformation. Oncotarget 2016, 7, 16636-16649, doi:10.18632/oncotarget.7674. 